\title{
Modeling and Diagnostics of Fuel Cell Porous Media for Improving Water Transport
}

\author{
E. F. Médicia ${ }^{\mathrm{a}}$ J. S. Allen ${ }^{\mathrm{a}}$ \\ aDepartment of Mechanical Engineering - Engineering Mechanics \\ Michigan Technological University, Houghton, Michigan 49931, USA
}

When a fuel cell is operating at high current density, water accumulation is a significant cause of performance and component degradation. Investigating the water transport inside the fuel cell is a challenging task due to opacity of the components, the randomness of the porous materials, and the difficulty in gain access to the interior for measurement due to the small dimensions of components. Numerical simulation can provide a good insight of the evolution of the water transport under different working condition. However, the validation of those simulations is remains an issue due the same experimental obstacles associated with in-situ measurements.

The discussion herein will focus on pore-network modeling of the water transport on the PTL and the insights gained from simulations as well as in the validation technique. The implications of a recently published criterion to characterize PTL, based on percolation theory, and validate numerical simulation are discussed.

\section{Introduction}

An increasing emphasis on modeling the behavior of liquid water in the porous media of proton exchange membrane (PEM) fuel cells has led to advances in understanding of water management as well as optimization of components for enhanced fuel cell water management. Yet there is much about transport of water, both liquid and vapor, within fuel cell porous media that remains difficult to model or simulate. As such, optimization of material properties for effective water transport remains largely an empirical effort.

There are two broad categories of modeling water transport in fuel cells. The first is a bulk model approach that is suitable for cell-level and system-level performance simulations. The calculations are relatively simple and can be optimized for computational efficiency, but the transport phenomena are reduced to a one-dimensional approximation. The second approach is a local model that is suitable for pore-level simulations of transport. The calculations are relatively slow, but provide physical insight into transport phenomena and how material property variations might alter transport. The local models tend to be too computationally intensive for any realistic integration into bulk models while the bulk models tend to oversimplify complex mass and energy transport. The latter is especially significant if multiphase transport occurs.

Pore-level models generally fall into two distinct methodologies. The first is based on a precise reconstruction of the porous material and then simulation of transport within a complex structure; often using a Lattice Boltzmann Method. The second methodology 
involves modeling transport phenomena accurately and simulating transport within a simplified structure. This latter approach often uses a network model of 'pores' and 'throats' to simulate the porous media structure. Both methodologies have merits and shortcomings. Also, both require characterization experiments that can be used to validate the simulation portion; transport in the first and structure in the second. These characterization experiments are a key element to pore-level modeling. The experiments need to be simple, yet representative of transport phenomena occurring in operating fuel cells.

The discussion herein will focus on pore-network modeling and the insights gained from simulations as well as the characterization experiments. Discussion will also include the accompanying characterization experiments necessary for validation in fuel cell porous media.

\section{Why the need for a local model?}

Localized flooding of PEM fuel cell components is widely recognized as a fundamental limit on performance and durability. Flooding may occur in the catalyst layer, the porous transport layer (PTL) ${ }^{1}$, and the flow field channels. Flooding in the PTL is thought initiate mass transport limitations that can lead to performance loss and component degradation. The presence of liquid water in the PTL reduces the area available for gas transport. Thus, there is a need to predict PTL saturation for a wide range of operating conditions and temperatures. In the absence of such predictive capability, many recent attempts at in-situ visualizing liquid water in the PTL have been conducted using NMR, X-ray tomography (2), neutron imaging $(3,4)$, and optical microscopy (5).

Saturation, or the fraction of porous media void space occupied by water, is traditionally correlated to injection pressure. In the absence of flow, the injection pressure can be related to the capillary pressure of specific size menisci. The pressure-saturation relationship and the Leverett function approximation are effective for prediction saturation of water in soils and porous rock where pressure is hydrostatic and constant. For very long times, the flow rate of water into the soil will decay to zero. Correlation between saturation and capillary pressure is essentially an equilibrium relationship. In contrast, fuel cells tend to operate in a constant flow rate condition and the pressure can vary to accommodate local conditions. The difference in the behavior of water transport between these to modes, constant pressure versus constant flow, is severe.

In an operating fuel cell, water will move through a subset of pores in the form of meandering rivulets. Typically, the water moves through the largest and/or the most wetting pores at approximately constant capillary pressure independent of the saturation level $(5,6)$. The uneven distribution of water is due to capillary effects destabilizing the liquid-gas interface resulting a flow pattern known as capillary fingering $(6,7)$. Uniform saturation is never observed with capillary fingering. As such, the pore size distribution has a more significant meaning in describing PTL water transport than does porosity or permeability. Since the liquid water distribution is uneven, the traditional continuum Darcy flow commonly used in porous media flows is inappropriate. Pore-level capillary pressure must be included in any model to predict water percolation within a PTL.

\footnotetext{
${ }^{1}$ Gas diffusion layer (GDL) is commonly used to describe both the PTL and combination of the PTL and microporous layer (MPL). See Mathias et al. (1) for a thorough discussion of low-temperature fuel cell porous media.
} 
The path taken by the liquid water through the PTL would appear to be stochastic and unpredictable. However, the behavior of the flow for any specific PTL can be modeled accurately and predictably.

\section{Pore-Network Model \& Simulation}

Soils and porous rock tend to have relatively uniform pore sizes and pore shapes that can be approximated as spherical. The wettability of water on these materials is generally uniform. In a PEM fuel cell PTL, however, there is nothing that resembles a 'pore' and the void space has a large range of confined volumes; with effective diameters from less than 5 $\mu \mathrm{m}$ to greater than $100 \mu \mathrm{m}$. The wide range of void length scales in a thin layer $(\sim 250 \mu \mathrm{m})$ results in poor connectivity between pores of similar size. Thus, for a pressure sufficient to drive water into a particular pore (void) size, not all pores of that size may receive water due to isolation (8).

The fiber arrangement in typical PEM fuel cell PTLs is such that relatively large void spaces are separated by small orifices, also known as 'throats', defined by crossing, nonwetting fibers. As such, the volume of water injected into a PTL during a saturation-pressure test may indicate many more small pores than are present. The pressure will be dictated by the opening between void spaces and the volume of water injected at that pressure will be determined by the void space. If a traditional interpretation of the pressure-volume data is used, the number of small pores corresponding to the high injection pressure will be grossly over-exaggerated. Any local model of water transport should be able to accommodate this heterogeneity, but this requirement does not mean that the heterogeneous structure be duplicated.

The network model was developed for the study of capillary flow regimes such as stable displacement, capillary fingering, and viscous fingering (7). One of the advantages of the network model is the low computational cost as compared with other methodologies such as Lattice Boltzmann Simulations (9). The network model has been shown to accurately simulate two-phase capillary flow in porous media (10). In the network model, the pores can be modeled as cylindrical $(7,11,12)$, spherical, or as a cylinder with variable cross sectional area. Pores can be arranged in regular lattice or using a unstructured arrangement (13). The uncertainty associated with the pore size has been assigned by defining a disorder parameter on a uniform pore size distribution $(11,12)$, by defining a pore size distribution (14), by a following a Weibull pore size distribution $(15,16)$, or by topographical reconstruction of the porous media (17).

The basic formulation of the network model includes a formulation for the variation of the capillary pressure as a function of the saturation at the pore level. The pressure-volume function may be constant $(7,11,12)$, may vary as a function of the meniscus position inside the pore mimicking pore throat effect (16), or by defining an irregular pore shape(15).

The network model has been used to simulate particular percolation scenarios such as oil recovery (18), drug delivery (19) water transport in soil(20), fuel cells (13, 16, 21-26), among others. In the case of modeling liquid water movement in fuel cell, this approach has been validated against data obtained in carefully designed percolation experiments on porous layers with similar wetting and morphological properties to PTLs $(27,28)$. Most recently, a novel validation criteria of the network simulation based on scaling of drainage in porous media has been developed (29).

The network model has been used as tool to access the effective permeability, diffusivity, and capillary pressure on the PTL, since it is difficult to determine them experimentally 
due to the nonuniform flow distribution $(15,21,22)$. This properties can later incorporated into a system-level fuel cell model. Another important issue related to the mass transport in fuel cell is evaporation. The network model has been extended to include evaporation in porous media in applications unrelated to fuel cells such us drying in soils (30-32). However there has been limited attempts to model evaporation in fuel cells using the network model (27).

\section{Simulation of Water Percolation in a PTL}

For this discussion, water transport in the PTL is simulated using a network model that consists of regular lattice of cylindrical tubes of constant length $l$ that represent the pores as illustrated in Figure 1. The tubes connect at nodes in groups of four for the two-dimensional network and in groups of six for the three-dimensional network. All pore volume in the network is contained within the tubes. The random sizes of the pores are simulated by generating tube radii based on a Weibull probability distribution function. The fluid flow behavior inside the network of tubes is modeled as modified Poiseuille flow that includes two-phase flow and capillary pressure effects (7). A detailed description of this model is presented in Medici and Allen (16).

The volumetric flow rate at a tube placed between the nodes ' $\mathrm{i}$ ' and ' $\mathrm{j}$ ', is defined as:

$$
q_{\mathrm{ij}}=\frac{\pi r_{\mathrm{ij}}^{4}}{8 \mu_{\mathrm{ij}}^{\mathrm{e}} l}\left(\Delta p_{\mathrm{ij}}-p_{\mathrm{ij}}^{\mathrm{c}}\right)
$$

where $r_{\mathrm{ij}}$ is the tube radius, $\Delta p_{\mathrm{ij}}$ is the pressure acting across the tube, and $p_{\mathrm{ij}}^{\mathrm{c}}$ is the capillary pressure in the tube when both phases are present. The volumetric flow rate definition is only valid when $\Delta p_{\mathrm{ij}}>p_{\mathrm{ij}}^{\mathrm{c}}$, otherwise $q_{\mathrm{ij}}=0$.

The effective viscosity within a pore, $\mu_{\mathrm{ij}}^{\mathrm{e}}$, is a function of the fluid position inside the tube $x_{\mathrm{ij}}$, the non-wetting (injected) fluid viscosity, $\mu_{\mathrm{nw}}$, and the wetting (displaced) fluid viscosity, $\mu_{\mathrm{w}}$. The effective viscosity is modeled to provide a smooth transition between the wetting and non-wetting viscosities while a pore is under saturated. When a tube is filled with only one fluid, $\mu_{\mathrm{ij}}^{\mathrm{e}}$ is equal the viscosity of that fluid.

The capillary pressure $p_{\mathrm{ij}}^{\mathrm{c}}$ is also modeled as a function of the fluid position within each tube. The capillary pressure is calculated using the average radius of the tubes intersecting at each node. As the meniscus moves toward the middle of a tube the capillary pressure is calculated using the radius of that tube. At the opposite end of the tube, capillary pressure is again calculated using the average radius of the intersecting. Capillary pressure varies smoothly between two nodes using cosine functions (equation 2) to mimic the throat effect at the ends of the pores.

$$
p_{\mathrm{ij}}^{\mathrm{c}}=\gamma \cos (\theta)\left[\left(1-\frac{r_{\mathrm{i}}}{2 \bar{r}_{\mathrm{i}}}-\frac{r_{\mathrm{j}}}{2 \bar{r}_{\mathrm{j}}}\right) \frac{1-\cos \left(2 \pi x_{\mathrm{ij}} / l\right)}{r_{\mathrm{ij}}}+\frac{1+\cos \left(\pi x_{\mathrm{ij}} / l\right)}{\bar{r}_{\mathrm{i}}}+\frac{1-\cos \left(\pi x_{\mathrm{ij}} / l\right)}{\bar{r}_{\mathrm{j}}}\right]
$$

where $\bar{r}_{\mathrm{i}}$ and $\bar{r}_{\mathrm{j}}$ are the average tube radius around node ' $\mathrm{i}$ ' and ' $\mathrm{j}$ ' respectively, $\gamma$ is the surface tension, and $\theta$ is the contact angle. The capillary pressure $p_{\mathrm{ij}}^{\mathrm{c}}$ is zero when the tube is filled with only one fluid. The normalized capillary pressure function for a random tube is shown in Figure 2. In this figure, the maximum capillary pressure is shown to be halfway between the nodes, but the maximum pressure may occur at any location between the nodes. 


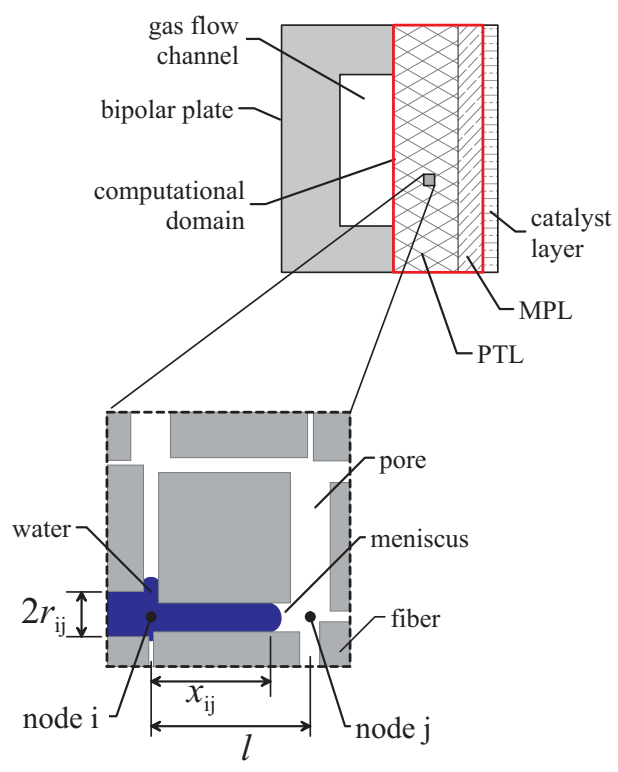

Figure 1: Pore-network domain. (16)

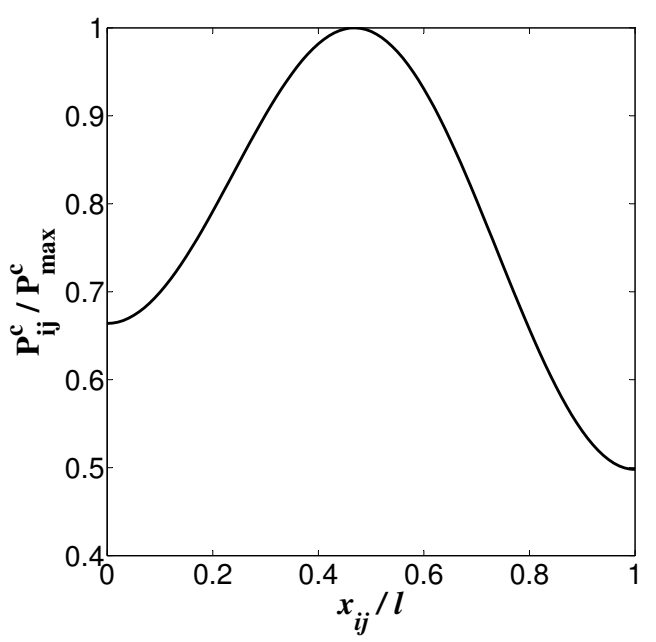

Figure 2: Normalized Internodal pressure function.

This form of the capillary pressure captures the effects of contact line pinning as a meniscus moves through the PTL. When a meniscus contact line pins, the meniscus may deform due to pressure thereby changing the mean curvature while remaining stationary. The advantage of using this approximation for the capillary pressure at a pore level is that it eliminates any need of any capillary pressure/saturation relationship or measurement such as the Leverett function.

Conservation of mass requires that the flow rate balance at each node for every time step in the simulation. The four flow rates at each note ' $i$ ' must sum to zero. Combining conservation of mass with the capillary pressure function and the viscous flow model results in an expression for the pressure balance at each node.

$$
\frac{\pi}{8 l} \sum_{\mathrm{j}=1}^{4} \frac{r_{\mathrm{ij}}^{4}}{\mu_{\mathrm{ij}}^{\mathrm{e}}}\left(\Delta p_{\mathrm{ij}}-p_{\mathrm{ij}}^{\mathrm{c}}\right)=0
$$

The unknown pressure gradient, $\Delta p_{\mathrm{ij}}$, can be solved by rearranging equation 3 . Note that the proportionality coefficient relating pressure drop to volumetric flow rate, in this case $\pi / 8$ for Poiseuille flow, is of no consequence to the solution. The model inputs include fluid properties, a distribution of pore sizes, and an effective contact angle. The latter two parameters are really describing a distribution of potential interface curvatures that generate the capillary back pressure. An equivalent network could be generated using constant diameter tubes and a distribution of wettability.

The resulting linear system of equations is solved using the Conjugated Gradient Stabilized Method (16). After calculating the pressure gradient, the new fluid position inside each tube is calculated proportional to the pressure gradient in that tube while satisfying mass conservation.

\section{Insights Gained from Isothermal Pore-Network Simulations}

For the results discussed in this paper, the length of the tubes, or pores, is fixed. for all the PTL simulations (15). The constant length of the pore may at first appear to be 

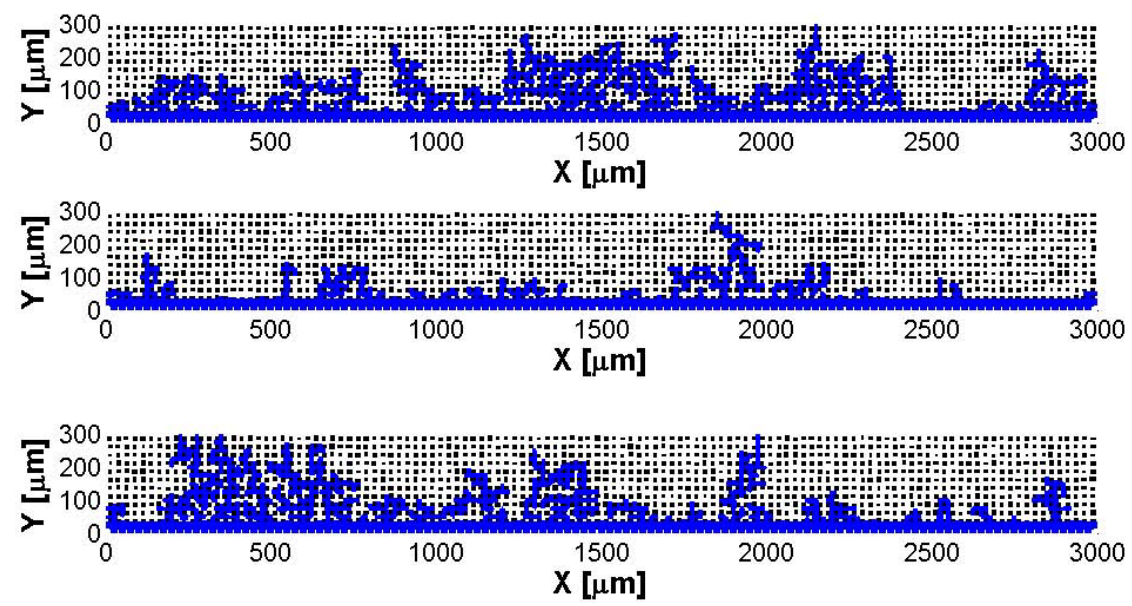

Figure 3. Water distributions at the time a capillary finger reaches the gas flow channel for three pore networks generated from the same pore size distribution (Weibull PDF, $\psi=5.25, k=3$ ). Simulations end times are 1192 seconds (top), 522 seconds (middle) and 1095 seconds (bottom). (16)
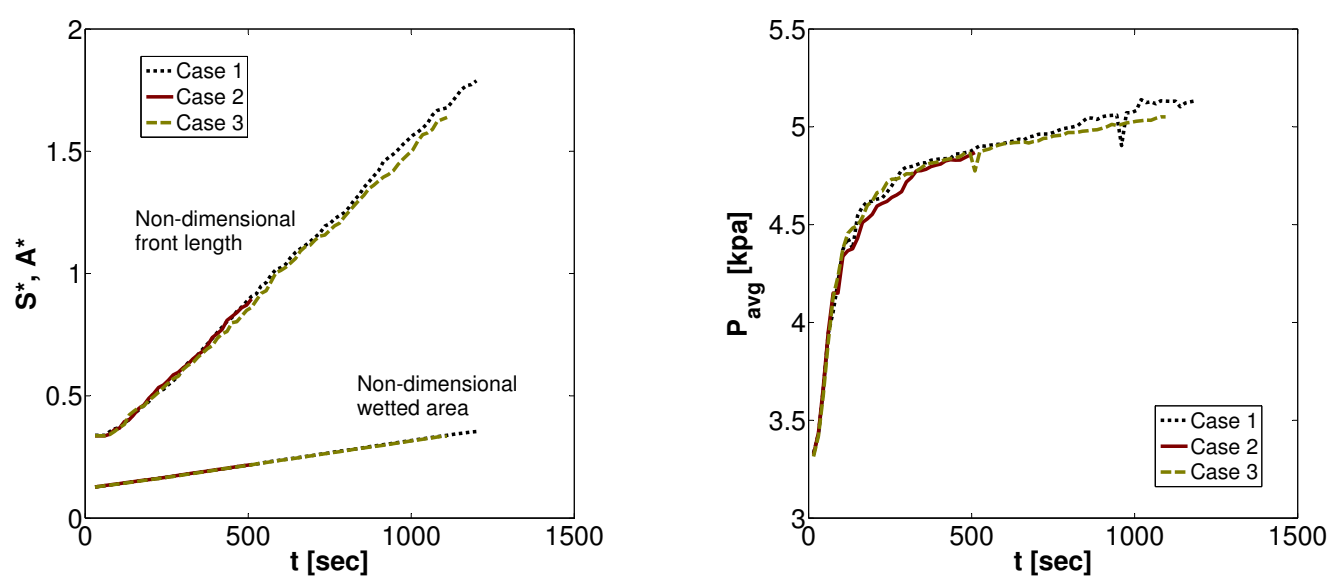

Figure 4. Non-dimensional measures of saturation and pressure in the PTL for the three network simulations shown in Figure 3. The simulation ends when the liquid water reaches the gas flow channel. (16)

a limitation of the network model, but the volume is compensated with an increase or decrease in the pore volume.

The stochastic nature of the pore arrangement in a PTL is accounted for by generating a random arrangement of pore sizes while maintaining a pore-size distribution that follows a Weibull probability distribution functions (PDF). The PDF used for the results presented herein was based on standard porosimetry data for Toray T090 PTL (15). The baseline contact angle value used in the simulations is $\theta=110$ degrees.

Observations based upon a single, randomized pore network may be misleading. An example would be a scenario where the randomly generated network has a series of large pores that are connected to one another spanning the PTL thickness. Therefore, fore each study three unique, random pore arrangements all of which follow the same pore size distribution using Weibull PDF are generated.

Water generated at the catalyst layer is modeled as a source term proportional to the cell current density and is distributed uniformly over the bottom nodes. This boundary 
condition is known to be overly simplistic (33), but is used for parametric studies of the effect of PTL properties on water transport. A Dirichlet boundary condition is used at the reactant flow channel with pressure equal to zero gauge pressure.

Figure 3 shows a two-dimensional simulation for the three pore-networks. The figures show the distribution of liquid water at the time that water reaches the gas flow channel. The results of this study are limited to isothermal liquid percolation without evaporation or reactant flow (16). The saturation, area occupied by water, is significantly different for each of the three pore-networks even though the networks all adhere to the same PDF. This observation of stochastic transport behavior is similar to ex-situ and in-situ experimental observations of water transport. Much of the apparent randomness of saturation is due to the thin section through which water is percolating. This apparent stochastic behavior of water transport in a PTL can be rectified through appropriate scaling.

Two measures of saturation are calculated during the percolation of water towards the gas flow channel. The first is the pore area occupied by water from a cross sectional view, referred to as the wetted area $A$. The second measure of saturation is the length of the interface between water and air, referred to as the front length $S$. These two time-dependent measures are used to quantify the water distribution inside the simulated PTL.

Figure 4a shows the non-dimensional front length $S^{*}$ and non-dimensional wetted area $A^{*}$ for the three pore networks plotted against simulation time. The front length, $S$, and the wetted area, $A$, are normalized by the domain perimeter and area, respectively. The nondimensional front length, $S^{*}$, and wetted area, $A^{*}$, collapse onto a single curve (Figure 4) despite a significant difference in PTL water content at the end of each simulation. The implication is that the transport of water is identical for each of the three pore networks, but time is an important factor. The stochastic nature of the pore arrangement within a distribution results in different times for water to reach the gas channel as indicated by the maximum values obtained for $S^{*}$ and $A^{*}$.

The average injection pressure $P_{\text {avg }}$ at the bottom of the PTL, which is effectively the catalyst layer, for each of the three simulations cases is plotted against time in Figure $4 \mathrm{~b}$. This average pressure can be understood as the pressure at which the water is injected into the PTL. Again, the time-dependent pressure is the same for all three pore-networks, but the rate of pressure change varies. The results of the three simulations shown in Figures 3 and 4 correspond to a Weibull PDF based on a Toray T060 PTL. Similar, but distinct, results can be obtained from PDF based on other types of PTLs. The average pressure curve is found to be similar to the pressure curve corresponding to a capillary fingering flow regime. An average injection pressure of 4.5 to $5 \mathrm{kPa}$, the maximum non-dimensional front length being approximately twice domain perimeter, and the maximum wetted area being a quarter of the total area agree with published data on capillary fingering percolation in PTLs $(6,34)$.

The self-similar behavior of water percolation in a PTL with the same distribution, but random arrangement of pores is a key finding. Other findings not discussed in detail include:

- Water transport in fuel cell porous media is not uniform and traditional capillary pressure - saturation functions can not accurately predict an effective permeability for a onedimensional reactant transport model. The balance between local menisci capillary pressure and viscous effects need to be considered.

- Water percolation in a heterogeneous porous material can be uniquely predicted through appropriate scaling and if time of flow is considered. 
- Pressure in the catalyst layer and water saturation in the PTL can be independently altered through variation in pore size distribution, wettability, and structure (16).

\section{Validation Experiments and Diagnostics}

The results and findings of pore network simulations of water percolation in fuel cell porous media are providing qualitative insight, but can these insights also be quantitative? The answer requires model validation. Unfortunately, there is no method for tracking pressure and flow at all locations of the gas-liquid interface. Therefore, any validation experiment should provide a measurable macroscopic response that is uniquely dependent on the local conditions.

An example of a unique macroscopic response to microscopic properties is the static contact angle. The contact angle is a macroscopic measurement of the shape of a liquid surface that is controlled by interfacial physics at the atomic level. Another example is a Leverett approximation for relating curvature distribution (local) and pressure (macro) in a porous media when there is no flow. The menisci curvatures are related to pressure and to pore sizes and wettabilities. A counter example is the use of a Leverett approximation for relating pressure (macro) and saturation (macro) when viscous effects are important at the local menisci level.

Validation experiments of pore-network simulations have often taken the form of a well-controlled model porous media in which the experimental pore structure looks nearly identical to the pore-network structure. Fuel cell porous media is anisotropic and heterogeneous. In order to validate the pore-network model for fuel cells, a simple ex-situ experiments is needed that can test the phenomena of interest; in this case, the balance between viscous stresses and capillary pressure on the percolation path.

A drainage experiment was used to investigate this balance. The experiment, described in detail in $(6,29,35)$, consists of compressing a fuel cell PTL between two platens. Water is injected in the center and flows radially towards the edges displacing air. An in-plane percolation study was chosen, as opposed to a through-plane percolation with the twodimensional simulations, in order to increase the time for observation. This arrangement is referred to as a pseudo Hele-Shaw experiment. The injection flow rate is fixed at various values and the measured time-dependent parameters are the wetted area and injection pressure.

For a fixed flow rate, the wetted area for any PTL sample in the pseudo Hele-Shaw test should be similar. There may be slight variation due to incomplete filling of the gap between the the platens, but the thin nature of the sample precludes this effect from being significant. The rate of water injection is characterized by the reference Capillary number based on the inlet condition.

$$
\mathrm{Ca}=\frac{Q \mu_{\mathrm{nw}}}{\sigma \pi r_{\mathrm{i}}^{2}}
$$

where $Q$ is the flow rate, $\mu_{\mathrm{nw}}$ is the non-wetting fluid (water) viscosity, $\sigma$ is surface tension and $r_{\mathrm{i}}$ is the inlet tube radius. Because the experiments are performed at a constant flow rate, the reference Capillary number depends only on the injection flow rate. The reference Capillary number should only be considered as dimensionless flow rate and is not indicative of the balance of forces at the moving gas-liquid interface. This latter balance is dependent upon position and time.

The time dependent parameters of pressure and wetted area can be scaled so as to reduce the drainage diagram to a single linear relationship that is unique to each PTL sample (29). 


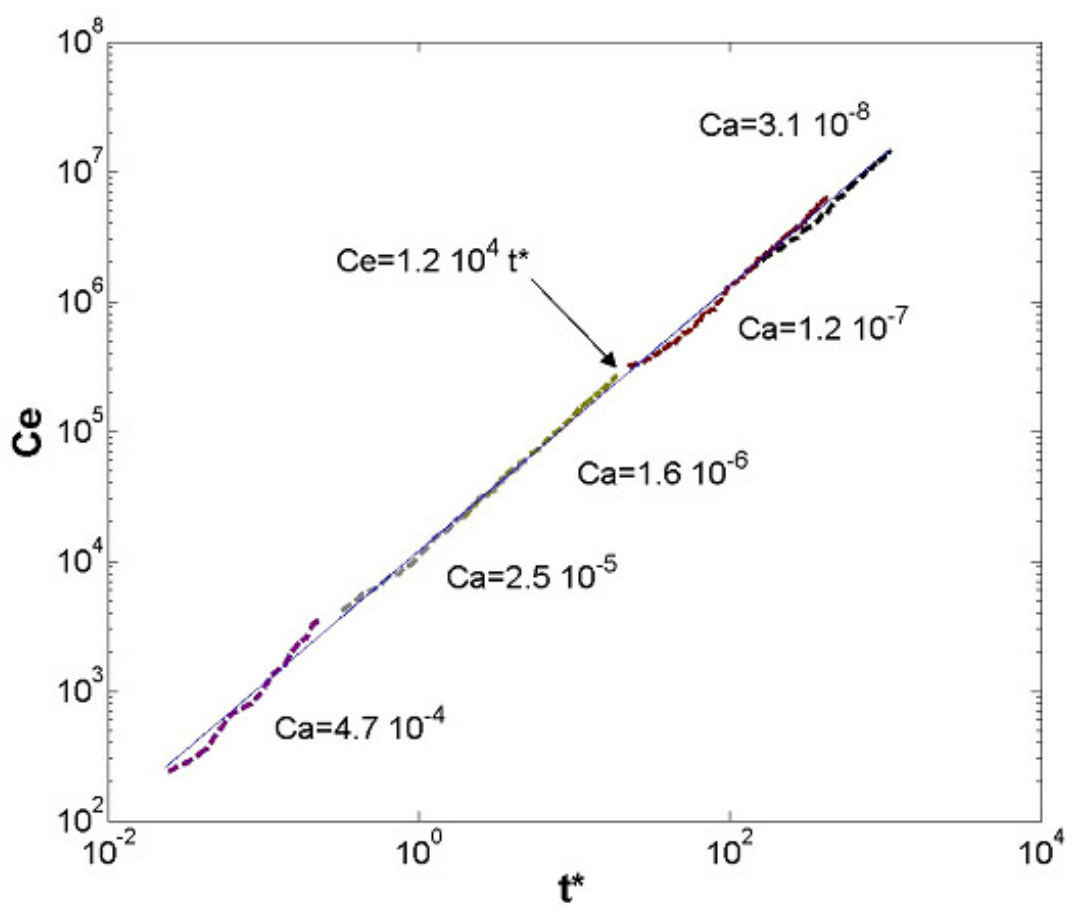

Figure 5. The solid line corresponds is a linear fit to the experimental data while the dashed lines correspond to experimental data for each capillary number.

The percolation pressure, the wetted area, and the injection flow rate scaled as a ratio of injection to dissipated energies. The injection energy is proportional to $P_{(t)} Q$, while the dissipated energy is proportional to $Q^{2} \mu l / h^{4}$. The ratio of these energies is defined as Ce:

$$
\mathrm{Ce}=\frac{P_{(t)} h^{3}}{\mu Q} \frac{A_{(t)}}{l^{2}}
$$

The term $A_{(t)} / l^{2}$ is a correction factor to compensate for the area cover by the water. In this scaling, $Q$ is the injection rate at the center of the sample. The injection pressure, $P(t)$, is equivalent to the percolation pressure and is a measure of the capillary pressure. The wetted area, $A(t)$, is a measure of the time-dependent saturation. The energy ratio, Ce, is time-dependent through the wetted area and the percolation pressure.

The time dependence of the percolation can also be scaled. The rate of percolation, in the pseudo Hele-Shaw experiments, is dependent upon the balance between capillary pressure and viscous stresses at the moving menisci. The form of the non-dimensional time $t^{*}$ is found to be:

$$
t^{*}=t \frac{\sigma h^{2}}{\mu l^{3}}
$$

where $\mu$ is the injected fluid viscosity and $\sigma$ is the injected fluid surface tension. Two length scales are used. The small length scale, $h$, is the sample thickness and the large length scale, $l$, is the sample size, which for this study is the diameter of the PTL sample. This time scale is, in effect, an inverse Capillary number based on local conditions as opposed to the inlet conditions previously discussed.

As the reference Capillary number is increased, the flow regime changes from stable displacement at small $t^{*}$ to capillary fingering at large $t^{*}$. The drainage flow patterns are 


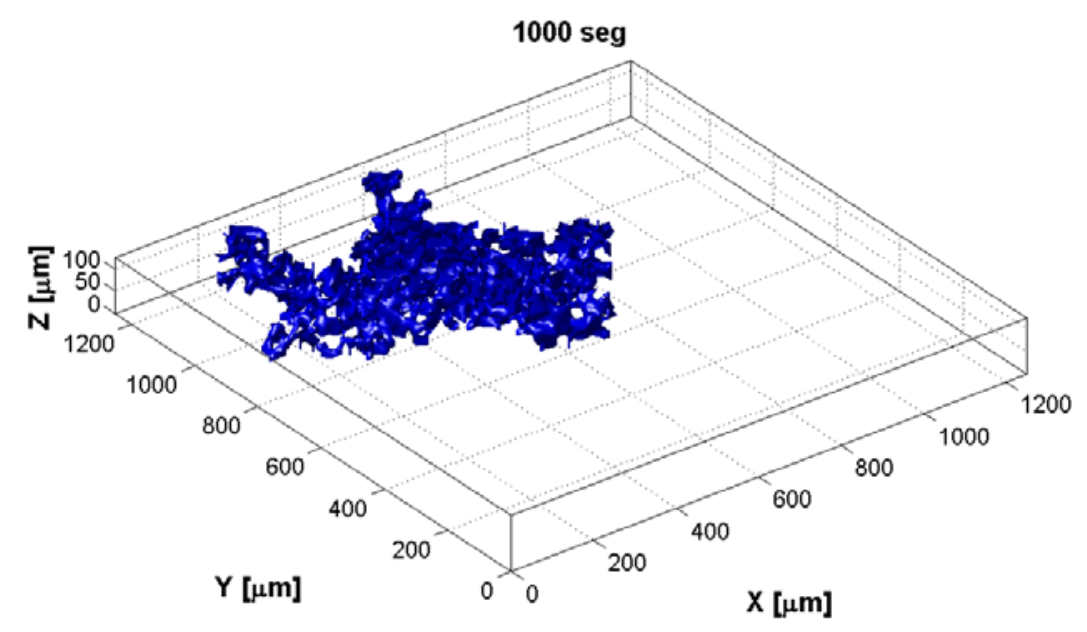

(a) isometric view of percolation

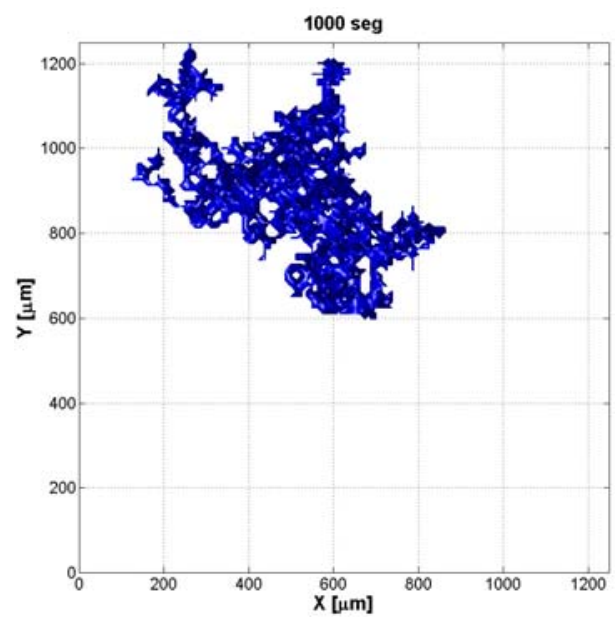

(b) top view - projected wetted area

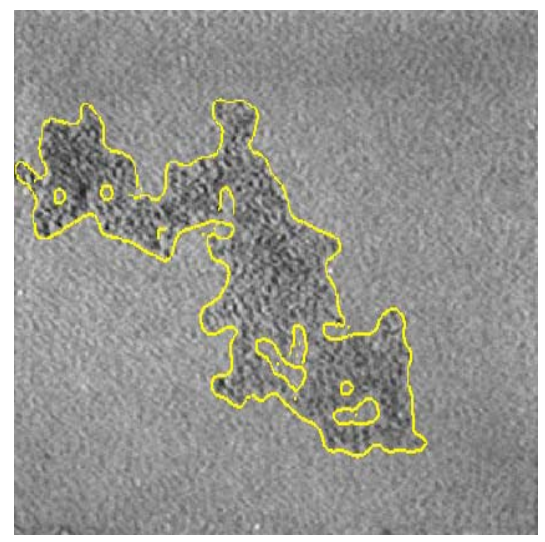

(c) experimental wetted area

Figure 6. Simulation of the pseudo Hele-Shaw experiment at $\mathrm{Ca}=6 \times 10^{-7}$ for a Weibull PDF corresponding to Toray T060 and a contact angle of 110 degrees. The time shown is at the end of the simulation when water reaches the edge of the sample. The experimental image of capillary fingering in a Toray T060 PTL is shown at the time that water has reached the sample edge. (36)

described in detail in Lenormand et al. (7). Transition between capillary fingering and stable displacement occurs at $t^{*}=1$ where viscous forces and capillary forces are balanced. As the transition between stable displacement and capillary fingering occurs the dissipated energy is reduced and the Ce increases. Similar, but unique Ce- $t^{*}$ relationships exist for other types of fuel cell PTLs, including nonwovens (29).

The energy ratio, Ce, relation to non-dimensional time, $t^{*}$, for different flow rates in a Toray T060 PTL (9\% PTFE wt) is shown in Figure 5. The flow rate for each test run is presented in the form of the reference Capillary number, $\mathrm{Ca}$, described by equation 4 . The local capillary number at each moving menisci will not be the same as the reference Ca. A linear regression fit through all of the experimental Ce is shown on a log-log scale. The curve shown in Figure 5 is generated from five separate tests performed at different reference Capillary numbers; i.e., different flow rates. Each test begins at time $t=0$, but the non-linear entrance effects at the onset of percolation are not shown (29).

Ideally, the Ce- $t^{*}$ relationship should have a slope is one and will remain so provided 


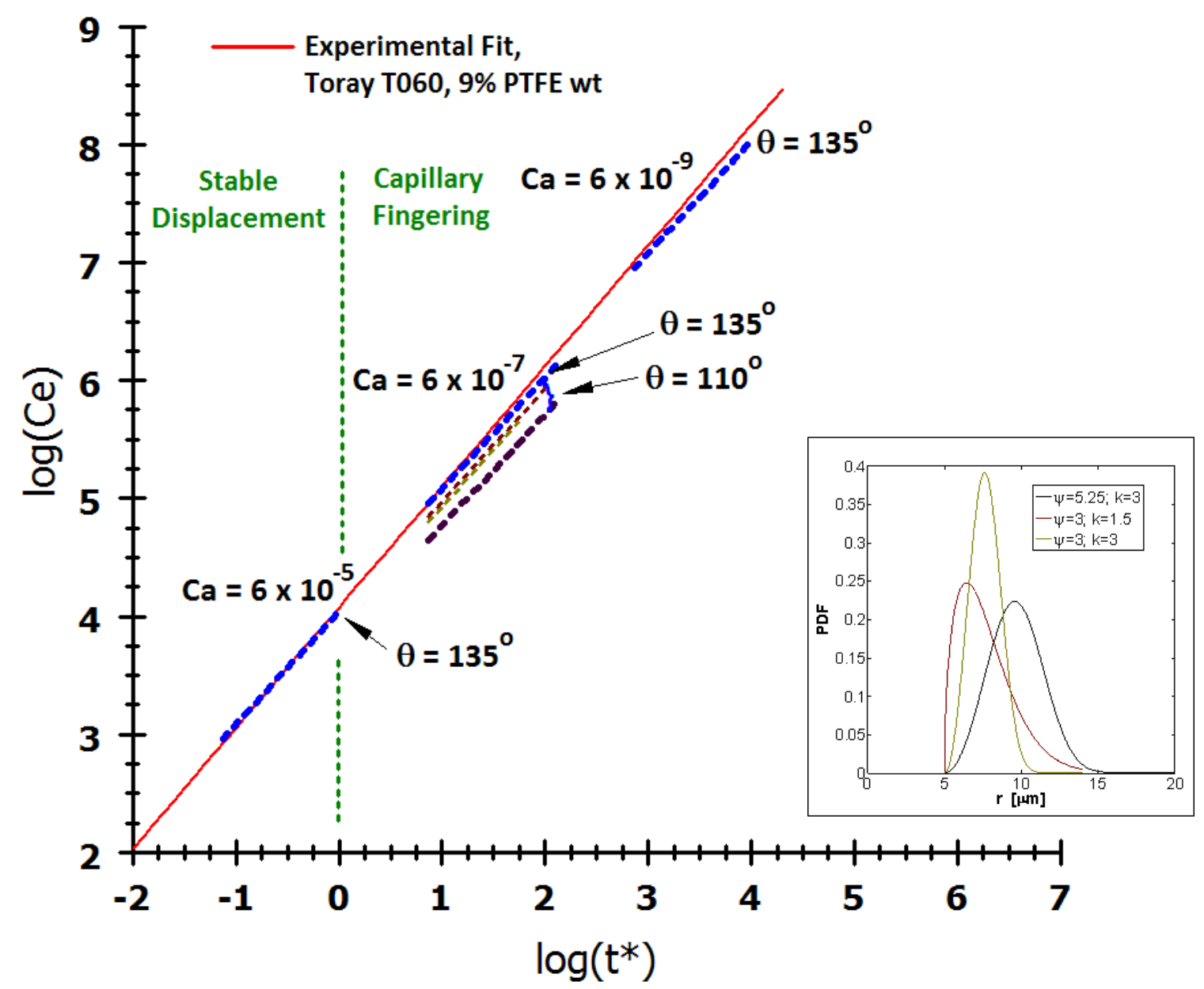

Figure 7. Numerical and experimental Ce- $t^{*}$ relations. Solid line corresponds to the fitting curve of the experimental results shown in Figure 5 while dashed lines correspond to numerical simulations. The contact angles used in every simulation are indicated with annotations. The colors of the numerical Ce match the colors of the Weibull distribution used to generate its pore size distribution.

there is no gas compression within the PTL during the test. If the injection flow rate exceeds the gas permeability of the sample, then energy will be stored via gas compression and the slope of the $\mathrm{Ce}-t^{*}$ relationship will be greater than one. The Toray data shown in Figure 5 exhibits an ideal behavior. Other low-PTFE loaded PTLs also have Ce- $t^{*}$ slopes of one, but the location of the curve is offset from the Toray data shown. The offset is due to variations in capillary pressure resulting from differences in morphology, structure, and wettability of the various PTLs (29).

This scaling and the pseudo Hele-Shaw experiment does not provide direct insight into water transport in fuel cells a priori. The scaling and experiments do, however, provide a method of validating the pore-network model in which the balance between viscous stress, capillary pressure, flow rate and saturation are being studied.

To that end, a three-dimensional simulation of the pseudo Hele-Shaw tests was conducted using the pore size distribution for the Toray T060 (15) as well as two additional Weibull PDFs for comparison. An initial effective contact angle of 110 degrees was used in the simulation with a reference Capillary number of $6 \times 10^{-7}$. Figure 6 shows an isometric and top view of the simulation for the pore size distribution corresponding to the Toray T060. The top view is equivalent to the experimental wetted area. 
The simulation results are compared to experimental results in Figure 7 using the Ce and $t^{*}$ scaling. The initial comparison is at $\mathrm{Ca}=6 \times 10^{-7}$ for the three pore size distributions. All three simulations are close to the experimental data, but the offset is the smallest for the Weibull PDF based on the Toray porosimetry data. Subsequent simulations used the Toray distribution and adjusted the contact angle to 135 degrees. With these parameters there is good agreement between the numerical simulations and the experimental data.

Once the proper offset and slope were obtained, two additional numerical simulations were performed at different reference capillary numbers to verify the validity of the scaling at extreme values. Capillary numbers of $6.0 \times 10^{-9}$ and $6.0 \times 10^{-5}$ were used for the capillary fingering and stable displacement simulations, respectively. The resulting energy ratio data, also plotted in Figure 7, are in good agreement with the experimental results in these ranges of capillary number.

At this point, the physics of water percolation through a Toray PTL has been fully captured in the pore-network model. In addition, the pseudo Hele-Shaw experiment used for validation can now be considered as a characterization test for fuel cell PTLs. However, there is much more to water transport in fuel cell PTLs than capillary pressure, flow rate, and viscous dissipation. Thermal and phase change effects have been conspicuously neglected and must be incorporated into a local model for a more realistic simulation and perspective of fuel cell water transport.

\section{Conclusions}

The traditional method of characterizing water saturation in a porous media is found to be ineffective due to the nature of the materials as well as the dynamics of percolation. Standard saturation-pressure data is implicitly presumes an equilibrium condition of constant pressure and no flow. In contrast, pressure can vary and there is effectively an constatn flow rate of water in operating fuel cells. These fuel cell conditions generate non-uniform water invasion of the porous layers due to a combination of capillary pressure and viscous stresses at the pore level.

Water percolation through fuel cell porous media is a complex process due to the anisotropic, heterogeneous nature of the materials. Yet, pore-level, or local, modeling of this flow can accurately capture what appears to be a stochastic process. The apparent stochastic behavior of water transport in a PTL can be simplified through appropriate scaling and careful experiment design.

The combination of a pore-network model, appropriately designed validation experiment, and scaling of key parameters demonstrates that the stochastic percolation of water in fuel cell porous media is unique to each material and can be predicted. Additional work is required to incorporate thermal effects and evaporation. The greater challenge lies in developing the necessary validation experiments that isolate these effects while providing a unique measurable macroscopic response to local conditions. 


\section{Acknowledgments}

This work was partially supported by the Department of Mechanical Engineering Engineering Mechanics at Michigan Technological University, U.S. Department of Energy contract DE-FG36-07GO17018 in collaboration with the Rochester Institute of Technology and General Motors, DOE contract DE-EE0000466 in cooperation with Ballard Power Systems, NSF grant CBET-078049, and with funds from the John F. and Joan M. Calder Endowed Associate Professor in Mechanical Engineering. The authors thank Dr. T. Trabold and J. Owegan at General Motors for providing PTL samples as well as for their technical support and to Mr. S. Stacy for the assistance with the drainage experiments.

\section{References}

1. M. Mathias, J. Roth, J. Fleming, W. Lehnert, in: Handbook of Fuel Cells - Fundamentals, Technology and Applications, Volume 3. W. Vielstich, A. Lamm, H. A. Gastiger, (eds.), John Wiley \& Sons, Chichester, UK, 517-537 (2003).

2. J. Eller, T. Rosén, F. Marone, M. Stampanoni, A. Wokaun, F. Büchi, J. Electrochemical Soc., 158, B963-B970 (2011).

3. Y.-S. Chen, H. Peng, D. S. Hussey, D. L. Jacobson, D. T. Tran, T. Abdel-Baset, and M. Biernacki, J. Power Sources, 170, 376-386 (2007).

4. M. C. Hatzell, A. Turhan, S. Kim, D. S. Hussey, D. L. Jacobson, and M. M. Mench, J. Electrochemical Soc., 158, B717-B726 (2011).

5. S. Litster, D. Sinton, and N. Djilali, J. Power Sources, 154, 95-105 (2006).

6. E. F. Médici and J. S. Allen, J. Power Sources, 191 417-427 (2009).

7. R. Lenormand, E. Touboul, and C. Zarcone, J. Fluid Mech., 189, 165-187 (1988).

8. C. M. Gribble, G. P. Matthews, G. M. Laudone, A. Turner, C. J. Ridgway, J. Schoelkopf, amd P. A. Gane, Chem. Eng. Sci., 66, 3701-3709 (2011).

9. K. Moriyama and T. Inamuro, Commun. Comput. Phys., 9, 1206-1218 (2011).

10. H. Vogel, J. Tolke, V. Schulz, M. Krafczyk, and K. Roth, Vadose Zone J., 4, 380 (2005).

11. J.-D. Chen and D. Wilkinson, Phys. Rev. Lett., 55, 1892-1895 (1985).

12. E. Aker, K. JØrgen MÅlØy, A. Hansen, and G. Batrouni, Trans. Porous Media, 32, 163-186 (1998).

13. J. Hinebaugh, Z. Fishman, and A. Bazylak, J. Electrochemical Soc., 157, B1651-B1657 (2010).

14. K. Stevenson, M. Ferer, G. Bromhal, J. Gump, J. Wilder, and D. Smith, Phys A: Stat. Mech. Appl., 367, 7-24 (2006).

15. J. T. Gostick, M. A. Ioannidis, M. W. Fowler, and M. D. Pritzker, J. Power Sources, 173, 277-290 (2007).

16. E. F. Médici and J. S. Allen, J. Electrochemical Soc., 157, B1505-B1514 (2010). 
17. V. Schulz, J. Becker, A. Wiegmann, P. Mukherjee, and C. Wang, J. Electrochemical Soc., 154, B419 (2007).

18. G. Pereira, W. Pinczewski, D. Chan, L. Paterson, and P. Øren, Trans. Porous Media, 24, 167201 (1996).

19. A. Stephanou, S. McDougall, A. Anderson, and M. Chaplain, Math. Comp. Mod., 44, 96-123 (2006).

20. J. Nieber, T. Bauters, T. Steenhuis, and J. Parlange, J. Hydrology, 231, 295-307 (2000).

21. K.-J. Lee, J. H. Nam, and C.-J. Kim, Electrochimica Acta, 54, 1166-1176 (2009).

22. B. Markicevic, A. Bazylak, and N. Djilali, J. Power Sources, 171, 706-717 (2007).

23. P. K. Sinha, P. P. Mukherjee, and C. Y. Wang, J. Mat. Chem., 17, 3089-3103 (2007).

24. P. K. Sinha and C.-Y. Wang, Electrochimica Acta, 52, 7936-7945 (2007).

25. P. P. Mukherjee, Q. Kang, and C.-Y. Wang, Energy Environ. Sci., 4, 346-369 (2011).

26. A. Z. Weber. R. Balliet, H. P. Gunterman, and J. Newman, in: Modern Aspects of Electrochemistry, M. Schlesinger (ed.), Springer, 273-416 (2009).

27. O. Chapuis, M. Prat, M. Quintard, E. Chane-Kane, O. Guillot, and N. Mayer, J. Power Sources, 178, 258-268 (2008).

28. A. Bazylak, D. Sinton, and N. Djilali, J. Power Sources, 176, 240-246 (2008).

29. E. F. Médici, J. S. Allen, Phy. Fluids, in review (2011).

30. M. Prat, Chem. Eng. J., 86, 153-164 (2002).

31. A. Yiotis, A. Stubos, A. Boudouvis, and Y. Yortsos, Adv. Water Res., 24, 439-460 (2001).

32. V. Surasani, T. Metzger, and E. Tsotsas, Int. J. Heat Mass Trans., 51, 2506-2518 (2008).

33. D. Harvey, J. G. Pharoah, and K. Karan, J. Power Sources, 179, 209-219 (2008).

34. J. Benziger, J. Nehlsen, D. Blackwell, T. Brennan, and J. Itescu, J. Membrane Sci., 261, 98106 (2005).

35. E. F. Médici and J. S. Allen, in Porous Media and Its Applications in Science, Engineering, and Industry - 3rd Int'l Conference, K. Vafai (ed.), 236-241, Montecatini, Italy (2010).

36. E. F. Médici, Ph.D. Dissertation, Michigan Technological University (2010). 